\title{
Concurrent Suppression of Integrin a5, Radixin, and RhoA Phenocopies the Effects of miR-31 on Metastasis
}

\author{
Scott Valastyan ${ }^{1,2}$, Amelia Chang ${ }^{1,2}$, Nathan Benaich ${ }^{1,3}$, Ferenc Reinhardt ${ }^{1}$, and Robert A. \\ Weinberg ${ }^{1,2,4}$ \\ ${ }^{1}$ Whitehead Institute for Biomedical Research, Cambridge, MA \\ 2Department of Biology, Massachusetts Institute of Technology, Cambridge, MA \\ ${ }^{3}$ Department of Biology, Williams College, Williamstown, MA \\ ${ }^{4}$ MIT Ludwig Center for Molecular Oncology, Cambridge, MA
}

\begin{abstract}
miR-31 inhibits breast cancer metastasis via the pleiotropic suppression of a cohort of prometastatic target genes that include integrin $\alpha 5$, radixin, and RhoA. We previously demonstrated that the concomitant overexpression of integrin $\alpha 5$, radixin, and RhoA was capable of overriding the anti-metastatic effects of ectopically expressed miR-31 in vivo. However, these prior studies failed to investigate whether the combined suppression of the endogenous mRNAs encoding these three proteins recapitulated the in vivo consequences of miR-31 expression on metastasis. We demonstrate here that shRNA-mediated concurrent downregulation of integrin $\alpha 5$, radixin, and RhoA is sufficient to phenocopy the full spectrum of described influences of miR-31 on metastasis in vivo, including this microRNA's impacts on local invasion, early post-intravasation events, and metastatic colonization. These findings provide mechanistic insights into the metastatic process and have implications concerning the importance of pleiotropy for the biological actions of microRNAs.
\end{abstract}

\section{Keywords}

miR-31; metastasis; breast cancer; microRNA; invasion-metastasis cascade; metastatic colonization

\section{INTRODUCTION}

Metastases are responsible for $90 \%$ of human cancer deaths and arise via a complex, multistep process termed the invasion-metastasis cascade $(1,2)$. In order to metastasize, cancer cells in a primary tumor must first acquire the capacity for motility, invade locally, intravasate into the systemic circulation, maintain viability during transit through the vasculature, extravasate into the parenchyma of a distant tissue, survive in this foreign microenvironment to form micrometastases, and finally re-initiate their proliferative program and establish macroscopic secondary tumors (metastatic colonization) (1). Metastatic colonization is the rate-limiting step of the invasion-metastasis cascade, yet relatively few molecular mediators of this process have been identified (2). 
MicroRNAs (miRNAs) are emerging as a class of critically important regulators of tumor metastasis. These evolutionarily conserved RNAs modulate gene expression at a posttranscriptional level via the pleiotropic suppression of sequence-complementary mRNA targets (3). A crucial role for miRNAs in tumor development has been firmly established by the identification of numerous miRNAs that function as bona fide oncogenes or tumor suppressor genes (4). Additionally, certain miRNAs have been more specifically implicated in the regulation of metastatic progression (5).

One such anti-metastatic miRNA is miR-31. We recently determined that miR-31 levels were inversely associated with the propensity for metastatic relapse in human breast carcinoma patients (6). Moreover, miR-31 expression was both necessary and sufficient to inhibit metastasis in human breast cancer xenografts (6). We attributed these effects to miR-31's capacity to intervene during at least three distinct steps of the invasion-metastasis cascade, doing so via the pleiotropic suppression of a cohort of pro-metastatic target genes (6). Subsequently, we discovered that the anti-metastatic consequences of ectopic miR-31 expression could be entirely reversed by the concomitant overexpression of three downstream effectors of this miRNA - integrin $\alpha 5$ (ITGA5), radixin (RDX), and RhoA (7).

Importantly, these earlier studies relied upon ectopic expression or overexpression of miR-31 and these target mRNAs, rather than modulation of the endogenous gene products. For this reason, we undertook to determine whether the concurrent suppression of the endogenous mRNAs encoding ITGA5, RDX, and RhoA was sufficient to phenocopy the impacts of ectopic miR-31 expression on metastasis. Success in this endeavor would indicate that these three proteins indeed function to promote metastasis and furthermore would implicate the pleiotropic suppression of ITGA5, RDX, and RhoA as a potential mechanism by which miR-31 antagonizes the metastatic phenotype.

\section{MATERIALS AND METHODS}

\section{Cell Culture, Plasmids, and Creation of Stable Cell Lines}

GFP-labeled MDA-MB-231 cells were described (6). SUM-159 cells were provided by S. Ethier, and cultured under conditions that we have delineated (6). miR-31 was expressed from pBABE-puro (6). Short hairpin RNAs (shRNAs) targeting the mRNAs encoding Luciferase, ITGA5, RDX, or RhoA were expressed from pLKO.1-puro (Open Biosystems); the sequences of these shRNAs hairpins are: shITGA5 \#3, CCACTGTGGATCATCATCCTA; shITGA5 \#4, CCTCAGGAACGAGTCAGAATT; shITGA5 \#5, CTCCTATATGTGACCAGAGTT; shRDX \#3, GCCAGAGATGAAACCAAGAAA; shRDX \#4, GCAGACAATTAAAGCTCAGAA; shRDX \#5, GCTAAATTCTTTCCTGAAGAT; shRhoA \#5,

GAAAGCAGGTAGAGTTGGCTT. Stable expression of the indicated plasmids was achieved via sequential retroviral or lentiviral transduction, followed by selection with puromycin (7). In the case of the Luciferase shRNA hairpin, target cells were subjected to either a single complete infection protocol ("shLuc" cells) or, alternatively, to three sequential complete infection protocols ("shLuc + shLuc + shLuc" cells); the latter strategy allowed us to obtain control cells containing approximately the same total number of shRNA molecules as were present in the shITGA5 + shRDX + shRhoA cells.

\section{Real Time RT-PCR}

Total RNA, including small RNAs, was isolated with a mirVana MicroRNA Isolation Kit (Ambion). RT-PCR-based detection of mature miRNAs and the 5S rRNA was achieved via utilization of a mirVana MicroRNA Detection Kit and gene-specific primer sets (Ambion). For detection of GAPDH, ITGA5, RDX, and RhoA transcript levels, cDNA was prepared 
from $500 \mathrm{ng}$ of total RNA using the SuperScript III First-Strand Synthesis System (Invitrogen), and subsequently quantified by SYBR Green real time RT-PCR (Applied Biosystems) using oligonucleotides that we have described previously (7).

\section{Immunoblotting}

Cell lysates were resolved by NuPAGE gel electrophoresis (Invitrogen), transferred to a PVDF membrane, and probed with antibodies recognizing $\beta$-actin (Santa Cruz), ITGA5 (Santa Cruz), RDX (Cell Signaling), or RhoA (Abcam).

\section{Invasion and Motility Assays}

In the invasion assays, $1.0 \times 10^{5}$ cells were seeded in Matrigel-coated chambers with $8.0 \mu \mathrm{m}$ pores (BD Biosciences); in the motility assays, $5.0 \times 10^{4}$ cells were plated atop uncoated membranes with $8.0 \mu \mathrm{m}$ pores (BD Biosciences). Cells were seeded in serum-free media, and then were allowed to translocate toward complete growth media for 20 hours. Noninvaded or non-migrated cells were then physically removed by scraping. Successfully translocated cells were subsequently visualized using a Diff-Quick Staining Set (Dade) and manually counted under a light microscope.

\section{Anoikis Assays}

Anoikis resistance was measured by seeding $7.5 \times 10^{4}$ cells in 6-well ultra-low attachment plates (Corning). After 24 hours, cells were resuspended in 0.4\% trypan blue (Sigma) and the proportion of viable cells was quantified using a hemocytometer.

\section{Measurements of in vitro Cell Proliferation}

Unless otherwise indicated, cellular proliferation was evaluated by seeding $1.0 \times 10^{5}$ cells per well in 6-well plates. Total cell number was assessed every two to three days by trypsinization and manual counting with a hemocytometer. Alternatively, proliferative kinetics were measured by seeding $5.0 \times 10^{2}$ cells per well in 96-well plates and then employing a CellTiter96 AQueous One Solution MTS Cell Proliferation Assay (Promega); cells were incubated with the MTS reagent for 1.5 hours, then total cell number was quantitated by measuring absorbance at $492 \mathrm{~nm}$ on a 96 -well plate reader.

\section{Xenograft Studies}

All animal studies complied with protocols approved by the MIT Committee on Animal Care. Age-matched NOD/SCID mice (propagated on-site) were employed in all xenograft experiments. For spontaneous metastasis assays, female mice were subjected to bilateral orthotopic injections into the mammary fat pads with $1.0 \times 10^{6}$ tumor cells resuspended in 1:2 Matrigel (BD Biosciences) plus normal growth media. For experimental metastasis assays, male mice were intravenously injected with $5.0 \times 10^{5}$ tumor cells (resuspended in PBS) via the tail vein. Lung metastasis was quantified at the indicated timepoints using a fluorescent dissecting microscope; these analyses were performed within three hours of specimen isolation. Tumor and lung histology was assessed by staining paraffin-embedded tissue sections with hematoxylin and eosin (H\&E). In our studies, metastatic foci less than $50 \mu \mathrm{m}$ in average diameter were classified as micrometastases; in contrast, macroscopic metastases were defined as metastatic lesions greater than $50 \mu \mathrm{m}$ in average diameter.

\section{Statistical Analyses}

Data are presented as mean \pm SEM from a representative experiment; each assay was independently repeated at least three times. Student's t-test was utilized for comparisons between groups, with $\mathrm{P}<0.05$ considered statistically significant. 


\section{RESULTS}

We previously demonstrated that the metastatic potential of human breast cancer xenografts could be potently suppressed by the ectopic expression of miR-31, and that the concomitant re-expression of three downstream effectors of this miRNA - ITGA5, RDX, and RhoA sufficed to override the anti-metastatic actions of miR-31 (6,7). These prior analyses relied, however, upon overexpression of ITGA5, RDX, and RhoA via viral expression vectors, rather than on modulation of the endogenous mRNAs encoding these proteins. Consequently, we wished to determine whether the simultaneous downregulation of endogenous ITGA5, RDX, and RhoA levels could phenocopy the effects of miR-31 expression on metastasis.

To this end, we created otherwise-metastatic MDA-MB-231 human breast cancer cells ("231 cells") that concurrently expressed shRNAs targeting the endogenous mRNAs encoding ITGA5, RDX, and RhoA. Multiple sequence-independent hairpins were tested for their efficacy in suppressing the targeted molecules (Supplementary Fig. S1 and data not shown). Importantly, cell lines generated upon sequential infection with several distinct combinations of shRNAs against these three proteins exhibited reductions in endogenous ITGA5, RDX, and RhoA levels reminiscent of the 50\%-60\% decreases in these three factors previously observed upon ectopic expression of miR-31 in 231 cells (6). Accordingly, we focused our subsequent analyses on those cell lines that concomitantly displayed between two- and three-fold suppression of ITGA5, RDX, and RhoA.

Non-specific, deleterious effects have been observed in cells expressing large quantities of shRNA molecules, ostensibly due to competition for shared components of the miRNA biogenesis machinery that might impair, in turn, the actions of a broad spectrum of important but otherwise functionally unrelated endogenous cellular miRNAs (8). Reassuringly, however, the processing of eight control miRNAs was unaffected in 231 cells simultaneously expressing ITGA5, RDX, and RhoA shRNAs (Supplementary Fig. S2). Hence, saturation of the miRNA biogenesis machinery was unlikely to have confounded our interpretations.

These shRNA-expressing 231 cells were first subjected to in vitro assays that gauge cellbiologic attributes required for metastasis. Coordinate suppression of ITGA5, RDX, and RhoA reduced invasion through an artificial extracellular matrix (Fig. 1A), cell motility (Fig. 1B), and resistance to anoikis-mediated cell death (Fig. 1C) in vitro. Among the different combinations of sequence-independent hairpins against ITGA5, RDX, and RhoA, the magnitude of the observed biological response correlated with the extent of knockdown achieved, suggesting that these outcomes arose as specific consequences of reduced levels of the targeted proteins. Importantly, concomitant suppression of ITGA5, RDX, and RhoA failed to alter in vitro proliferation kinetics (Supplementary Fig. S3), indicating the absence of significant cytostatic or cytotoxic effects due to the expression of these shRNAs. Together, these data confirmed that ITGA5, RDX, and RhoA control in vitro behaviors critical for the acquisition of metastatic competence.

While the preceding experiments demonstrated that the concurrent suppression of ITGA5, RDX, and RhoA impaired metastasis-relevant phenotypes in 231 cells in vitro, the consequences of concomitantly inhibiting these three proteins on the in vivo behavior of carcinoma cells remained unclear. Accordingly, we determined whether the simultaneous suppression of ITGA5, RDX, and RhoA impaired in vivo metastasis in a manner comparable to that triggered by ectopic miR-31 expression. In order to do so, we orthotopically implanted into the mammary fat pads of mice 231 cells expressing either shRNAs against the mRNAs encoding these three factors or, alternatively, a miR-31 expression vector. 
Consistent with our prior findings $(6,7)$, miR-31 enhanced primary mammary tumor growth by 1.5 -fold; in contrast, the combined shRNA-evoked suppression of ITGA5, RDX, and RhoA failed to affect primary tumor size (Fig. 2A).

After normalizing for differences in primary mammary tumor growth, miR-31-expressing 231 cells formed $95 \%$ fewer lung metastases than did controls in this assay; similarly, the coordinate shRNA-conferred knockdown of ITGA5, RDX, and RhoA inhibited the incidence of pulmonary metastatic lesions by $95 \%$ (Fig. 2B). Cells concurrently expressing additional combinations of shRNAs against alternative complementary sequences in the three targeted mRNAs yielded identical results, implying that these effects were attributable specifically to the ability of these shRNAs to reduce the levels of ITGA5, RDX, and RhoA (Supplementary Fig. S4). Hence, the concomitant downregulation of endogenous ITGA5, RDX, and RhoA levels closely phenocopied miR-31-imposed inhibition of metastasis in this xenograft assay.

Remaining unresolved, however, were the particular step(s) of the invasion-metastasis cascade that were impaired due to the combined suppression of ITGA5, RDX, and RhoA in 231 cells. In our previous work, we observed that miR-31 impinges on three distinct steps of the invasion-metastasis cascade in vivo: local invasion, one or more early post-intravasation events (intraluminal viability, extravasation, and/or initial survival in distant tissues), and metastatic colonization $(6,7)$. Consequently, we evaluated whether the coordinate shRNAmediated suppression of ITGA5, RDX, and RhoA was sufficient to recapitulate one or more of miR-31's multiple effects on various discrete steps of the metastatic process.

To assess potential impacts on local invasion, we examined the histopathological appearance of orthotopically implanted primary mammary tumors. As before (6,7), control 231 cells formed invasive primary tumors, while miR-31 expression resulted in primary mammary tumors with a well-encapsulated phenotype (Fig. 3A). Cells containing shRNAs directed against ITGA5, RDX, and RhoA formed non-invasive primary mammary tumors that were indistinguishable at a histopathological level from the tumors generated by miR-31expressing cells (Fig. 3A and Supplementary Fig. S5A). Therefore, the concurrent suppression of ITGA5, RDX, and RhoA phenocopied the in vivo influences of miR-31 on local invasion.

Possible effects on early post-intravasation events were investigated by quantifying 231 cells in the lungs one day after intravenous injection via the tail vein. As anticipated $(6,7)$, miR-31-expressing cells were five-fold impaired in terms of their ability to persist in the lungs at one day post-injection; cells with coordinately suppressed ITGA5, RDX, and RhoA levels owing to the simultaneous expression of shRNAs against these three mRNAs were also five-fold less prevalent than controls at this timepoint (Fig. 3B and Supplementary Fig. S5B). These outcomes were not attributable to differing abilities of the cells to become lodged initially in the lung microvasculature, as equal numbers of cells from each group were detected in the lungs 10 minutes after intravenous injection (Supplementary Fig. S6). Instead, these observations indicated that the combined inhibition of ITGA5, RDX, and RhoA phenocopied the effects of miR-31 on early post-intravasation events in the lungs in vivo.

To evaluate putative effects on metastatic colonization, the sizes of subsequently arising lung metastases in intravenously injected mice were assessed at three months postimplantation. As expected (6,7), whereas control 231 cells generated robust macroscopic lung metastases, miR-31-expressing cells formed only small micrometastases (Fig. 3C). Similarly, cells containing shRNAs concomitantly targeting ITGA5, RDX, and RhoA failed to spawn macroscopic metastases and generated only small micrometastases (Fig. 3C and 
Supplementary Fig. S5C). Hence, the simultaneous suppression of ITGA5, RDX, and RhoA phenocopied the in vivo consequences of miR-31 expression with respect to metastatic colonization.

In this same intravenous injection assay, miR-31-expressing 231 cells formed $95 \%$ fewer lung metastases than did controls, while the concomitant shRNA-mediated suppression of ITGA5, RDX, and RhoA decreased the number of pulmonary metastatic lesions by $90 \%$ (Fig. 4 and Supplementary Fig. S7). Together, the preceding experiments indicated that the concurrent shRNA-mediated suppression of endogenous ITGA5, RDX, and RhoA levels was sufficient to phenocopy the full spectrum of miR-31's described influences on in vivo metastasis.

It remained possible that the ability of concomitantly suppressed ITGA5, RDX, and RhoA to phenocopy miR-31's anti-malignant actions arose due to some peculiarity of 231 cells. To address this, we extended our analyses to SUM-159 human breast cancer cells. Like 231 cells, SUM-159 cells are highly aggressive in vitro and exhibit reduced invasiveness, motility, and anoikis resistance upon ectopic miR-31 expression (6). We created SUM-159 cells that concurrently expressed shRNAs targeting the endogenous mRNAs encoding ITGA5, RDX, and RhoA, again utilizing multiple sequence-independent hairpins against an individual transcript (Supplementary Fig. 8A). Of note, sequential infection with several distinct combinations of shRNAs against these three proteins resulted in reductions in endogenous ITGA5, RDX, and RhoA levels quite similar to the 50\%-60\% decreases in these three factors elicited by ectopic miR-31 expression in aggressive human breast cancer cells (6). The simultaneous knockdown of ITGA5, RDX, and RhoA failed to alter the in vitro proliferative kinetics of SUM-159 cells (Supplementary Fig. 8B), thus excluding potential confounding effects related to this parameter. Consistent with our observations in 231 cells, the concomitant suppression of ITGA5, RDX, and RhoA in SUM-159 cells impaired several in vitro surrogate markers of metastatic capacity, namely invasiveness through an artificial extracellular matrix (Supplementary Fig. 8C), cell motility (Supplementary Fig. 8D), and anoikis resistance (Supplementary Fig. 8E). Thus, the ability of the simultaneous shRNAconferred suppression of ITGA5, RDX, and RhoA to phenocopy the consequences of ectopic miR-31 expression on various cell-biologic attributes required for metastasis was not confined to 231 cells.

\section{DISCUSSION}

In this report, we provide evidence that the concomitant downregulation of the endogenous levels of ITGA5, RDX, and RhoA substantially impairs the in vivo metastatic capacity of otherwise-aggressive human breast cancer xenografts. Perhaps surprisingly, our data indicate that even a relatively modest change in the levels of these three proteins can profoundly impact the metastatic potential of human mammary carcinoma cells in vivo. In consonance with these findings, other laboratories have previously described positive associations between elevated levels of ITGA5, members of the RDX family, or RhoA in carcinoma cells and disease progression in human tumors (9-11).

Notably, the simultaneous suppression of ITGA5, RDX, and RhoA impedes not only the initial escape of neoplastic cells from a primary tumor, but also the ability of alreadydisseminated cancer cells to thrive at distant organ sites. In light of the fact that significant numbers of disseminated tumor cells are frequently already present in the systemic circulation, bone marrow, and/or distant organs of human carcinoma patients even at early stages of disease progression $(12,13)$, ITGA5, RDX, and RhoA may represent attractive therapeutic targets owing to their actions in metastasis-promotion at secondary organ sites. In particular, the capabilities of these proteins to alter metastatic colonization efficiency may 
be quite significant, as completion of this rate-limiting step of the invasion-metastasis cascade is believed to dictate disease outcome in many human cancers (1).

miR-31 is predicted to regulate $>200$ mRNAs $(14,15)$. The principal finding of the present study, which indicates that miR-31's anti-metastatic effects can derive largely - if not entirely - from the ability of this miRNA to suppress a cohort of only three downstream target genes, are therefore quite surprising. Nonetheless, our data do not preclude the existence of still-uncharacterized miR-31 target genes that impinge upon the metastatic process in a manner that is functionally masked by the consequences of altering ITGA5, RDX, or RhoA levels. Also, our observation that the simultaneous suppression of ITGA5, RDX, and RhoA fails to recapitulate miR-31's ability to enhance primary mammary tumor growth suggests that additional miR-31 downstream effectors can mediate miR-31dependent influences on the in vivo behavior of carcinoma cells that are mechanistically unrelated to metastasis.

Collectively, our observations have implications concerning the pathogenesis of high-grade malignancies. The present data indicate that miR-31 sits atop a regulatory pathway that affects multiple steps of the metastatic process, altering both the capacity of cancer cells to exit from a primary tumor and the ability of already-disseminated neoplastic cells to survive and thrive in the foreign microenvironment afforded by the site of metastasis. As such, miR-31's pleiotropic anti-metastatic capabilities appear to position this miRNA as a critical safeguard against the acquisition of metastatic competence. Consequently, it is plausible that therapeutic strategies directed toward restoring miR-31 function may prove to be clinically useful in limiting metastatic progression in human carcinomas.

\section{Supplementary Material}

Refer to Web version on PubMed Central for supplementary material.

\section{Acknowledgments}

We thank Julie Valastyan for critical reading of this manuscript; Matthew Saelzler, Lynne Waldman, and other members of the Weinberg lab for helpful discussions; and the MIT Koch Institute Histology Facility for tissue sectioning. This research was supported by the NIH (RO1-CA078461), MIT Ludwig Center for Molecular Oncology, U.S. Department of Defense, and Breast Cancer Research Foundation. S.V. is a U.S. Department of Defense Breast Cancer Research Program Predoctoral Fellow. R.A.W. is an American Cancer Society Research Professor and a Daniel K. Ludwig Foundation Cancer Research Professor.

\section{REFERENCES}

1. Fidler IJ. The pathogenesis of cancer metastasis: the 'seed and soil' hypothesis revisited. Nat Rev Cancer. 2003; 3:453-8. [PubMed: 12778135]

2. Gupta GP, Massagué J. Cancer metastasis: building a framework. Cell. 2006; 127:679-95. [PubMed: 17110329]

3. Bartel DP. MicroRNAs: target recognition and regulatory functions. Cell. 2009; 136:215-33. [PubMed: 19167326]

4. Ventura A, Jacks T. MicroRNAs and cancer: short RNAs go a long way. Cell. 2009; 136:586-91. [PubMed: 19239879]

5. Valastyan S, Weinberg RA. MicroRNAs: crucial multi-tasking components in the complex circuitry of tumor metastasis. Cell Cycle. 2009; 8:3506-12. [PubMed: 19838065]

6. Valastyan S, Reinhardt F, Benaich N, et al. A pleiotropically acting microRNA, miR-31, inhibits breast cancer metastasis. Cell. 2009; 137:1032-46. [PubMed: 19524507]

7. Valastyan S, Benaich N, Chang A, Reinhardt F, Weinberg RA. Concomitant suppression of three target genes can explain the impact of a microRNA on metastasis. Genes Dev. 2009; 23:2592-7. [PubMed: 19875476] 
8. Grimm D, Streetz KL, Jopling CL, et al. Fatality in mice due to oversaturation of cellular microRNA/short hairpin RNA pathways. Nature. 2006; 441:537-41. [PubMed: 16724069]

9. Sanchez-Carbayo M, Socci ND, Lozano J, Saint F, Cordon-Cardo C. Defining molecular profiles of poor outcome in patients with invasive bladder cancer using oligonucleotide microarrays. J Clin Oncol. 2006; 24:778-89. [PubMed: 16432078]

10. McClatchey AI. Merlin and ERM proteins: unappreciated roles in cancer development? Nat Rev Cancer. 2003; 3:877-83. [PubMed: 14668818]

11. Sahai E, Marshall CJ. Rho-GTPases and cancer. Nat Rev Cancer. 2002; 2:133-42. [PubMed: 12635176]

12. Nagrath $\mathrm{S}$, Sequist LV, Maheswaran $\mathrm{S}$, et al. Isolation of rare circulating tumour cells in cancer patients by microchip technology. Nature. 2007; 450:1235-9. [PubMed: 18097410]

13. Hüsemann Y, Geigl JB, Schubert F, et al. Systemic spread is an early step in breast cancer. Cancer Cell. 2008; 13:58-68. [PubMed: 18167340]

14. Krek A, Grün D, Poy MN, et al. Combinatorial microRNA target predictions. Nat Genet. 2005; 37:495-500. [PubMed: 15806104]

15. Grimson A, Farh KK, Johnston WK, Garrett-Engele P, Lim LP, Bartel DP. MicroRNA targeting specificity in mammals: determinants beyond seed pairing. Mol Cell. 2007; 27:91-105. [PubMed: 17612493] 


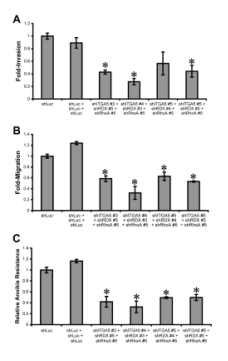

Figure 1. Simultaneous Suppression of ITGA5, RDX, and RhoA Impairs Metastasis-Relevant Traits in vitro

(A) Invasive capacity of 231 cells through Matrigel in a Boyden chamber transwell assay. $\mathrm{n}$ =3. (B) Transwell motility assays employing 231 cells. $n=3$. (C) Sensitivity of 231 cells to anoikis-mediated cell death after 24 hours of suspension culture. $n=3$. Luc $=$ Luciferase. Asterisks: $\mathrm{P}<0.05$ relative to shLuc + shLuc + shLuc. Data are presented as mean \pm SEM from a representative experiment; each assay was independently repeated at least three times. 


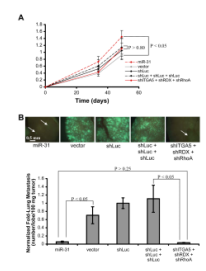

Figure 2. Concomitant Suppression of ITGA5, RDX, and RhoA Phenocopies miR-31-Evoked Inhibition of Spontaneous Metastasis in vivo

(A) Primary mammary tumor growth kinetics upon orthotopic implantation of 231 cells. The experiment was terminated after seven weeks due to primary tumor burden. $n=4$. (B)

Fluorescent images of murine lungs to visualize 231 cells 49 days after orthotopic injection (top panels). Quantification of metastatic burden (bottom panel). Arrows: micrometastases. $\mathrm{n}=4$. Luc = Luciferase. shRNAs utilized: shITGA5 \#5, shRDX \#5, and shRhoA \#5. Data are presented as mean \pm SEM from a representative experiment; each assay was independently repeated three times. 


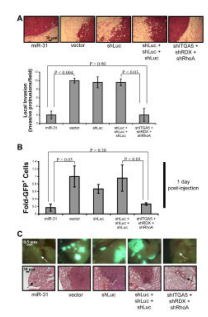

Figure 3. Concurrent Knockdown of ITGA5, RDX, and RhoA Phenocopies the Influences of miR-31 on Local Invasion, Early Post-Intravasation Events, and Metastatic Colonization in vivo (A) H\&E staining of 231 cell primary mammary tumors 34 days after orthotopic implantation (top panels). Quantification of local invasion (bottom panel). $n=4$. (B) Prevalence of 231 cells in the lungs one day after intravenous introduction. $n=4$. (C) Fluorescent images of murine lungs to visualize 231 cells 84 days after tail vein injection (top panels). H\&E staining of lungs from animals implanted with the indicated tumor cells (bottom panels). Arrows: micrometastases. $n=5$. Luc $=$ Luciferase. shRNAs utilized: shITGA5 \#5, shRDX \#5, and shRhoA \#5. Data are presented as mean \pm SEM from a representative experiment; each assay was independently repeated three times. 


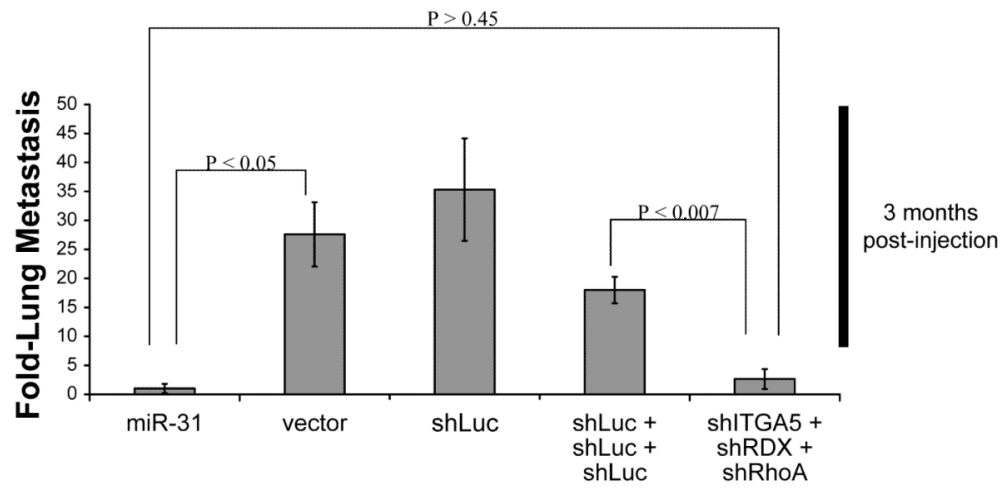

Figure 4. Simultaneous Suppression of ITGA5, RDX, and RhoA Phenocopies miR-31-Mediated Inhibition of Experimental Metastasis in vivo

Lung metastatic burden 84 days subsequent to intravenous injection of the indicated 231 cells. $\mathrm{n}=5$. Luc $=$ Luciferase. shRNAs utilized: shITGA5 \#5, shRDX \#5, and shRhoA \#5. Data are presented as mean \pm SEM from a representative experiment; this assay was independently repeated three times. 